ఠ

Open Access Full Text Article

REVIEW

\title{
Advantages, limitations, and diagnostic accuracy of photoscreeners in early detection of amblyopia: a review
}

This article was published in the following Dove Press journal:

Clinical Ophthalmology

22 July 2016

Number of times this article has been viewed

Irene Sanchez ${ }^{1,2}$

Sara Ortiz-Toquero ${ }^{1,2}$

Raul Martin ${ }^{2,3}$

Victoria de Juan ${ }^{2,4}$

'Department of Theoretical Physics, Atomic and Optics, School of Optometry, ${ }^{2}$ Optometry Research Group, IOBA-Eye Institute, University of Valladolid, Valladolid, Spain; ${ }^{3}$ Faculty of Health and Human Sciences, Plymouth University, Plymouth, UK; ${ }^{4}$ Department of Ophthalmology, Hospital Ramón y Cajal, Madrid, Spain

Correspondence: Victoria de Juan Department of Ophthalmology, Hospital Ramón y Cajal, Carretera de Colmenar Viejo, km 9.100, 28034 Madrid, Spain Tel +34 983 I8 4853

Email victoriadejuanryc@gmail.com
Abstract: Amblyopia detection is important to ensure proper visual development and avoid permanent decrease of visual acuity. This condition does not produce symptoms, so it is difficult to diagnose if a vision problem actually exists. However, because amblyopia treatment is limited by age, early diagnosis is of paramount relevance. Traditional vision screening (conducted in $<3$ years) is related with difficulty in getting cooperation from a subject to conduct the eye exam, so accurate objective methods to improve amblyopia detection are necessary. Handheld devices used for photoscreening or autorefraction could offer advantages to improve amblyopia screening because they reduce exploration time to just few seconds, no subject collaboration is needed, and they provide objective information. The purpose of this review is to summarize the main functions and clinical applicability of commercially available devices for early detection of amblyopia and to describe their differences, advantages, and limitations. Although the studies reviewed are heterogeneous (due to wide differences in referral criteria, use of different risk factors, different types of samples studied, etc), these devices provide objective measures in a quick and objective way with a simple outcome report: retest, pass, or refer. However, due to major limitations, these devices are not recommended, and their use in clinical practice is limited.

Keywords: handheld photoscreener, photoscreening, binocular autorefraction, amblyopia screening

\section{Introduction}

Objective vision screening is of paramount importance in the early detection of amblyopia to minimize the visual impact characterized by decreased visual acuity without ocular structural disorder. ${ }^{1}$ This condition is the most common cause of monocular vision impairment in children $<10$ years of age, ${ }^{2}$ affecting $2 \%-5 \%$. $^{3-5}$

Several causes can provoke amblyopia such as defocused images (eg, anisometropia), deprivation (eg, infantile cataracts), or misaligned eye images (eg, constant strabismus). ${ }^{6}$ Amblyopia may not produce obvious symptoms, and it is common that a family may be unaware that a vision problem exists until their child grows and verbally communicates or when the child starts intense visual activities, such as school work. ${ }^{2}$ Amblyopia treatment is limited by age (visual maturation period) $;^{7}$ for this reason, early diagnosis is of paramount importance to start the treatment as soon as possible. ${ }^{8}$ Moreover, strabismus has been reported to affect $\sim 4 \%$ of children, ${ }^{9,10}$ and this condition is highly related with amblyopia. However, small strabismus or micro-strabismus is not easily detected by non-health care professionals (family, parents, or teachers at the kindergarten). 
Traditional vision screening in children $<3$ years can be difficult due to poor collaboration during the exam (visual acuity, cover test, refraction, Hirschberg test, Bruckner reflex, etc). ${ }^{11}$ For this reason, devices used for photoscreening or autorefraction ${ }^{10,12}$ offer some advantages over traditional vision chart-based assessment, for example, by reducing time and providing specific visual information ${ }^{5}$ useful to detect some risk factors for amblyopia (strabismus, anisometropia, etc). The American Academy of Pediatrics, the American Academy of Ophthalmology, the American Association for Pediatric Ophthalmology and Strabismus (AAPOS), and the American Association of Certified Orthoptists ${ }^{12}$ recommend the use of different photoscreening devices for amblyopia detection in preschool population.

Photorefraction was developed as a screening tool to rule out amblyogenic ametropias in children. ${ }^{13-15}$ Photorefractors use an infrared camera that captures and analyzes images of the red reflex of undilated pupil assessing the correct alignment of both eyes and estimating the eye refractive error. ${ }^{12}$ These images can be evaluated by eye care practitioners and reading centers, or using automatic software. Effectiveness of photorefractive devices has been tested for detection of anisometropia, hyperopia, myopia, and astigmatism. Besides, some devices include further analysis of the eye misalignment, ptosis, or lens opacity. ${ }^{16}$ Photoscreening is a progress in preschool exploration because it is fast, simple, and little cooperation of the child is required. ${ }^{12}$

Several studies have been conducted to establish a photoscreening program guideline, such as the Lions Clubs of Tennessee $(1998)^{17}$ or Vision in Preschoolers (VIP) study $(2007)^{9}$ that enrolled volunteers children in preschools. The objective of these programs was to resolve deficiencies previously recognized in photoscreening programs which were related with the high referral rates, variable interpretation, or low positive predictive values.

Meanwhile, an important advance in photoscreeners technology in pediatric population assessment is the development of handheld devices. Handheld devices permit to avoid problems related with the chinrest and forehead position. There are different reports on use of handheld devices in photoscreening for preschool children, such as the Lions Clubs International Foundation ${ }^{17}$ where $\sim 250,000$ children were screened using handheld photoscreeners. Other batterypowered devices, for example, the SureSight autorefractor or Spot photoscreener, have showed ${ }^{18}$ great applicability in reducing the number of children who did not receive the recommended preschool vision exam in settings with high prevalence of astigmatism (Native American, in particular) ${ }^{19}$ or rural populations. ${ }^{20-23}$
The purpose of this review is to summarize the main functions and clinical applicability of different handheld binocular photoscreeners and to describe their differences, advantages, limitations, and accuracy of their results in amblyopia screening.

\section{Methods}

An extensive electronic search of the MEDLINE and PubMed databases using individual and combinations of keywords (photoscreening, photoscreener, plusoptix, powerrefractor, binocular autorefraction) was conducted to identify the relevant articles published in English language until January 2016. Additional references (from different sources) identified through the literature cited in the selected articles were included as well. In summary, 92 publications were identified. The search results were refined, and publications related with tabletop devices, accommodation, and myopia control studies were excluded. Only reports focused on handheld devices and studies focused on early detection of amblyopia (with indicators like sensitivity, specificity, repeatability, and reproducibility) with clear description of the referral criteria were finally included.

\section{Results}

Eleven photorefraction and photoscreener devices were identified and included in this review.

\section{Referral criteria}

The AAPOS referral criteria guidelines for detection of risk factors for strabismus or amblyopia are as follows: ${ }^{23}$

- Anisometropia (spherical or cylindrical) $>1.5 \mathrm{D}$.

- Any manifest strabismus.

- Hyperopia $>3.5 \mathrm{D}$ in any meridian.

- Myopia magnitude $>3.0 \mathrm{D}$ in any meridian.

- Any media opacity $>1 \mathrm{~mm}$ in size.

- Astigmatism $>1.5 \mathrm{D}$ within $10^{\circ}$ or $90^{\circ}$ or $180^{\circ}$, or $>1.0 \mathrm{D}$ in oblique axis.

- Ptosis with $<1 \mathrm{~mm}$ margin reflex distance.

Different criteria have been proposed to improve the screening outcomes. Some devices follow the AAPOS referral criteria; however, other devices use different referral criteria, for example, Rowat criteria, VIP criteria, ${ }^{2}$ manufacturer criteria, ${ }^{20,24}$ or their own referral criteria. ${ }^{9,23}$ The lack of uniformity in referral criteria comparison between devices and studies difficult. ${ }^{25}$

\section{Refraction screening techniques}

Automated infrared photorefraction is a potentially useful technique that works on the principle of eccentric 
Table I Overview of the main characteristics of the photoscreeners

\begin{tabular}{|c|c|}
\hline \multicolumn{2}{|c|}{ Photorefraction characteristics } \\
\hline Measurement distance & One meter in front of the child \\
\hline Illumination & Scotopic \\
\hline Correction & Without correction \\
\hline Fixation stimuli & Flashes lights or noises \\
\hline Measurement time & $<5$ seconds \\
\hline Pupil state & Undilated \\
\hline \multicolumn{2}{|c|}{ Patient will be referred if the outcomes suggest: } \\
\hline \multicolumn{2}{|c|}{ Significant refractive error } \\
\hline \multicolumn{2}{|l|}{ Anisocoria } \\
\hline \multicolumn{2}{|l|}{ Anisometropia } \\
\hline \multicolumn{2}{|c|}{ Pupillary centers discrepant $>10^{\circ}$} \\
\hline \multicolumn{2}{|l|}{ Ptosis } \\
\hline
\end{tabular}

photorefraction. It allows determining the refractive state of the eye by assessing a video image of the eye pupil. ${ }^{21}$ Autorefraction is another method used in vision screening. It involves infrared light either with automated skiascopy methods or with a similar technology that allows obtaining the eye refraction automatically. ${ }^{12}$

All photorefraction devices are used for analyzing pupil size and shape; assessment of the pupil size, crescent location, red reflex symmetry, and corneal light reflex determining if the explored eye has significant hyperopia, myopia, astigmatism, amblyopia, or strabismus. Myopic crescents appear in the flash direction, whereas hyperopic crescents show the opposite behavior. ${ }^{22}$ Some general characteristics of the photoscreeners are summarized in Table 1.

\section{Photoscreeners}

\section{MTI photoscreener}

The MTI photoscreener (Medical Technology Industries, LLC, Riviera Beach, FL, USA or Medical Technology and Innovations Inc, Lancaster, PA, USA) was the first of several devices used for detection of amblyopia which do not require subject cooperation. ${ }^{17,22}$ The manufacturer and Donahue introduced some modifications in light crescent interpretation, firstly in pupil size measurement and then in assessment of light crescent from the posterior pupil margin. ${ }^{22}$

This off-axis photoscreener utilizes two photographs taken with an eccentric flash that rotates $90^{\circ}$ for each pair of images. ${ }^{10}$ The photographs should be taken in mesopic light conditions, in patients with undilated pupils and no spectacle correction. ${ }^{10}$ The two images are captured on Polaroid film or Fuji 3000B film. ${ }^{7,22}$ The photograph should be interpreted by an expert examiner (reading center) who can assess the presence of amblyogenic factors, such as high hyperopia, strabismus, media opacity, or anisometropia, ${ }^{7,22}$ and classify the photograph as pass or fail. If, after three attempts, no adequate photographs are obtained or if the image is unreadable, the case is classified as a fail and may be referred for a complete eye examination. ${ }^{2}$

The major limitation or disadvantage of the MTI system is that photographs show a high degree of variability and their interpretation necessitates an expert (reading center), and thus, photographic interpretation is not immediately possible. ${ }^{7}$

\section{PowerRefractor}

PowerRefractor (Plusoptix, Nuremberg, Germany) began to be used since 2000s to determine refraction and for amblyopic screening. ${ }^{26}$ This device uses an eccentric infrared light with two-flash photorefractometers to measure the eye refraction. Briefly, a PowerRefractor assesses the slopes of the brightness distributions in the patient pupil and converts it into refractive error (in a spherical range of -7.00 to $+5.00 \mathrm{D}$ ). Previously, it was necessary to individually calibrate the refraction achieved with trial lenses in a standard fashion.

This device uses "six-armed retinoscopes" with six infrared light emitting diode (LED) arrays (placed around an infrared digital video camera) to determine the refractions sequentially in the $30^{\circ}, 90^{\circ}$, and $150^{\circ}$ pupil meridians in both eyes simultaneously. Opposite blocks of LEDs illuminate each pupil meridian two times and create crescents in the pupil. ${ }^{16,25}$ The device also determines the pupil size and the angular position of the pupil axis (determined by centering the first Purkinje image in the pupil, with a resolution of $0.9^{\circ}$ ). The axis of measurement is controlled with a gaze tracker. The patient's fixation is directed toward the camera, and measurements are achieved continuously. The refraction is displayed in red or green color on the device display. Red color signals represent an uncertain result, whereas green represent precise values. ${ }^{16,26}$

This instrument is very useful to quantify the dynamics of the oculomotor system because it is a completely open-field refractor which can measure pupil response, pupil diameter, eye gaze position, and lag of accommodation. ${ }^{16,25,27}$

\section{PowerRefractor II}

The PowerRefractor II (Plusoptix) provides some advantages over PowerRefractor including three operating modes: the "gaze scan" mode permits measurement and visualization of the fixation angle and/or the strabismus deviation; the "full scan" mode allows determining full binocular refraction and measuring pupil size; and the third mode, the "dynamic scan" mode, allows obtaining measurements of temporal changes in pupil size and accommodation. ${ }^{21}$ This device has the advantage of continuous recording of accommodation and pupil size and position of both eyes, improvement in 
the PowerRefractor outcomes, and repeated measurement of eye refraction. ${ }^{28}$ However, it does not allow the possibility of using individual calibrations. ${ }^{21}$

\section{Plusoptix}

A series of the Plusoptix (Plusoptix) photoscreeners have been designed for vision screening in children and have been approved by the US Food and Drug Administration (FDA) as a refractor. However, these devices have not been widely employed in pediatric primary health care. ${ }^{4}$ These instruments are designed with a smiling face on the camera that automatically uses lights and warble sounds to draw the child's attention to the camera. ${ }^{411,13,29}$ Refractive status is determined by assessing the distribution of the reflected light across the pupil. The measurement range is -7.0 to $+5.0 \mathrm{D}$ in steps of $0.25 \mathrm{D}$ for spheres and cylinders, and $4.0-8.0 \mathrm{~mm}$ in steps of $0.1 \mathrm{~mm}$ for pupil diameter. ${ }^{4,5,11,13,23,29,30}$

The Plusoptix photoscreener series involve five different devices, Plusoptix CR03, Plusoptix S04, Plusoptix S08, Plusoptix S09, and Plusoptix A09, with slight differences between them.

- Plusoptix CR03 is the second-generation photorefractive device, and is able to quantify the refractive error in pupils with diameter over $3.00 \mathrm{~mm}$. The Plusoptix CR03 and the PowerRefractor II are similar devices with slight differences in camera hardware. ${ }^{11}$ Both consist of an infrared digital video camera mounted on a flat-screen monitor operated by a computer. Around the lens, there are six blocks of infrared LEDs. A circling light in the camera is used to direct the child's attention, and in a rapid succession, three meridians are illuminated, and measures are taken. The patient's eye is shown in real time, and refractive results are calculated automatically. Measurements are taken continuously, and the display on the monitor allows the examiner to accept or reject measurements according to the color-coded reliability displayed. ${ }^{11}$

- Plusoptix S04 photoscreening camera is the thirdgeneration PowerRefractor II. ${ }^{29}$ This device uses infrared light with a coaxial video camera to measure pupil diameter, pupil distance, ocular alignment, and refractive error (with or without cycloplegia) in both eyes simultaneously. ${ }^{29}$ It is necessary to be connected with a computer, which analyzes the captured images and provides the outcome report on screen immediately. Image acquisition time averages between 5 and 10 seconds. $^{23,29}$ Plusoptix S04 photoscreener is an FDA-approved handheld, user-friendly, binocular, infrared photoscreener that is useful for screening risk factors for amblyopia. ${ }^{31}$ The values of sensitivity and specificity depend on the referral criteria used. For example, following manufacturer criteria, a positive predictive value of $78 \%$, a sensitivity of $98 \%$, and a specificity of $69 \%$ have been proposed. ${ }^{3}$ However, the AAPOS modified these criteria and found a positive predictive value of $90 \%$, a sensitivity of $98 \%$, a specificity of $88 \%$, and a false-negative rate of $1.5 \%{ }^{3}$ Besides, these results are influenced by the child age. ${ }^{23}$ Table 2 summarizes the accuracy indexes for detecting risk factors for refractive amblyopia.

- Plusoptix S08 is the third-generation model and is able to measure eye refractive status with undilated pupils. ${ }^{5}$ This device uses an infrared video camera to measure the refractive status of both eyes using eccentric photorefraction. Refractive status is not provided in cases of poor fixation, ocular misalignment $>10^{\circ}$, or eye pathology, and in these cases, the device recommends "refer option". Furthermore, if the refractive status is outside of the measurement range, the outcome displayed is just "hyperopia" or "myopia".

The manufacturer's referral criteria depend on the child age which is classified into four groups, 6-12, 13-36, 37-72, and 73-240 months, with different values of sensitivity and specificity. For each age group, the manufacturer provides referral criteria with five possible diagnostics: anisometropia, astigmatism, myopia, hyperopia, and anisocoria. ${ }^{3}$

- The Plusoptix S09 and A09 photorefractors designed in 2001 are third-generation models of the original Plusoptix, ${ }^{13}$ which are used with infants older than 6 months of age with 0.8 second of acquisition time. ${ }^{30}$ The Plusoptix A09 photorefractor is marketed as an autorefractor for eye care practice, whereas the S09 is marketed as a pediatric vision screener. ${ }^{13,31}$

In binocular measurement mode, Plusoptix S09 and A09 photoscreeners do not provide refraction of both eyes in the presence of significant strabismus. ${ }^{4}$ Refraction is provided in $60.1 \%$ of attempts. ${ }^{13}$ Moreover, these devices cannot measure pupil distance and diameter in patients with $>10^{\circ}$ of ocular misalignment. In these situations, it is necessary to use monocular measurement mode. If the spherical equivalent is out of the range, the measurement value only displays "hyperopia" or "myopia" providing only a cylindrical estimated value. ${ }^{4,13}$

These devices showed a sensitivity of $44.4 \%$ and specificity of $97.7 \%$ for hyperopia detection; however for myopia detection the sensitivity was $85.7 \%$ and specificity of $94.7 \%{ }^{4}$ Moreover strabismus detection showed a sensitivity of $40.7 \%$ and specificity of $98.3 \%$ (Table 2$)^{4}$

\section{iScreen}

The iScreen (iScreen Vision Inc, Cordova, TN, USA) photoscreener was introduced in 2000. It is a handheld device, 
Table 2 Accuracy indexes detecting risk factors for refractive amblyopia with different commercial devices in comparison with traditional exploration or other devices

\begin{tabular}{|c|c|c|c|c|c|c|c|c|}
\hline Device & Sensitivity (\%) & Specificity (\%) & $\begin{array}{l}\text { Data } \\
\text { success (\%) }\end{array}$ & $\begin{array}{l}\text { Accuracy } \\
\text { rate (\%) }\end{array}$ & PPV (\%) & NPV (\%) & FP (\%) & FN (\%) \\
\hline MTI & $81^{22}$ & $81^{22}$ & $94^{2}$ & $81^{22}$ & - & - & - & - \\
\hline \multicolumn{9}{|l|}{ PowerRefractor } \\
\hline \multicolumn{9}{|c|}{ PowerRefractor II } \\
\hline Plusoptix CR03 & $97.9^{11}$ & $70.6^{11}$ & - & - & $97.3^{11}$ & $75^{\prime \prime}$ & - & - \\
\hline \multirow{2}{*}{ Plusoptix S04 } & $98^{3}$ & $88^{3}$ & - & - & $90^{3}$ & - & - & $1.5^{3}$ \\
\hline & $99^{23}$ & $82^{23}$ & - & - & $86^{23}$ & - & $18^{23}$ & $1.2^{23}$ \\
\hline \multirow[t]{3}{*}{ Plusoptix A09 } & $89^{18}$ & $80^{18}$ & - & - & - & - & - & - \\
\hline & $94.9^{4}$ & $67.5^{4}$ & - & - & - & - & - & - \\
\hline & $85^{24}$ & $73^{24}$ & - & $82^{24}$ & $78^{24}$ & $81^{24}$ & - & - \\
\hline \multirow[t]{2}{*}{ Plusoptix SI2 } & $100^{35}$ & $61^{35}$ & $54^{35}$ & - & $76^{35}$ & $100^{35}$ & - & - \\
\hline & $91^{24}$ & $71^{24}$ & - & $84^{24}$ & $81^{24}$ & $85^{24}$ & - & - \\
\hline iScreen & $87^{22}$ & $76^{22}$ & - & $81^{22}$ & - & - & - & - \\
\hline Spot & $78^{24}$ & $59^{24}$ & - & $74^{24}$ & $74^{24}$ & $65^{24}$ & - & - \\
\hline Spot vI.0.3 & $89^{1}$ & יו & - & - & - & - & - & - \\
\hline \multirow[t]{3}{*}{ Spot v2.0.16 } & $87.7^{14}$ & $75.9^{14}$ & $91^{14}$ & - & $48^{14}$ & $96^{14}$ & - & - \\
\hline & $92.6^{20}$ & $90.6^{20}$ & - & - & $58.1^{20}$ & $98.9^{20}$ & - & - \\
\hline & $96^{35}$ & $87^{35}$ & $97^{35}$ & - & $90^{35}$ & $94^{35}$ & - & - \\
\hline Spot vI.I5I & $88.1^{14}$ & $71.9^{14}$ & - & - & - & - & - & - \\
\hline \multirow[t]{2}{*}{$2 \mathrm{WIN}$} & $7 I^{24}$ & $67^{24}$ & - & $71^{24}$ & $76^{24}$ & $62^{24}$ & - & - \\
\hline & $73^{24}$ & $76^{24}$ & - & $76^{24}$ & $80^{24}$ & $67^{24}$ & - & - \\
\hline
\end{tabular}

Notes: Different referral criteria might be used in each instrument. The values of Plusoptix CR03 are accuracy indexes for detection of cylindrical power with cyclopegic retinoscopy and those of Spot v2.0.16 are the accuracy indexes for detection of refractive error.

Abbreviations: PPV, positive predictive value; NPV, negative predictive value; FP, false positive; FN, false negative.

smaller than the MTI photoscreener, and weighs $\sim 2.5$ pounds ( 3.5 pounds lighter than the MTI). This device is an off-axis binocular photoscreener that captures a single image that is electronically transmitted to a reading center for analysis. The first-generation iScreen was a tabletop device. The iScreen Vision Screener 3000, introduced in 2011, has been significantly redesigned and miniaturized. ${ }^{22}$

It captures two-eye photos in rapid succession in two axes with a separation of $90^{\circ}$. The individual perceives only one flash before a blink (speed of image acquisition is similar to the MTI), which requires flash rotation before a second image could be acquired. The images can be reviewed immediately on the device, and even if the child is not fixing at the camera, a second set of images can be acquired immediately. Suitable images are securely transferred to the manufacturer from the device via Internet $^{22}$ which are then analyzed. A secure email is returned with the results classified as pass, pass/monitor, refer, or retest. ${ }^{22}$

The iScreen currently allows the technician to acquire the images and retake inadequate images, but if the original image is deleted, it cannot be recovered. Only a pair of images per child could be sent to be evaluated. The iScreen does not allow acquiring and sending multiple images. ${ }^{22}$

\section{Spot}

The Spot (PediaVision, Lake Mary, FL, USA or Welch Allyn, Skaneateles Falls, NY, USA) vision screener was introduced in 2011 as a successor of the SureSight (handheld monocular photoscreener). ${ }^{13}$ It is a handheld wireless, battery-powered device, weighs 2.55 pounds, and is easily portable with a computer interface. It captures a picture and measures binocular noncycloplegic refractive error, ocular alignment, pupil size, and pupil distance using the optical reflex. This information is displayed on a 4.5-inch touch screen, and stored for printing and generating Excel database for analysis. PediaVision allows measuring the refraction in a range of $\pm 7.50 \mathrm{D}$, with accuracy between +0.25 and $-0.50 \mathrm{D}$ for spherical refractive error and between +0.50 and $-1.00 \mathrm{D}$ for cylindrical power. Referral criteria can be manually designed (including the cutoff values for myopia, hyperopia, and anisometropia), and the device provides a dichotomic result like pass or fail which is instantly displayed on the screen. Results could be sent wirelessly to a printer. All data are stored on the device and can be transferred to a computer for storage and analysis using wireless or a portable data storage device. ${ }^{13,15,32}$

This device will not record data until the image is properly focused, and provide visual clues to assist in image-capturing process. Images can be obtained rapidly with multiple readings in three different meridians in just 2 seconds, and the device shows one of the several messages: "all measurements within range", "complete eye exam recommended", "pupils too small", "pupils not found", "out of range", or "continue attempting to obtain a reading". $14,20,32$ 
The instrument uses the operator cutoff values to make a referral proposal in significant refractive error, anisometropia, anisocoria, or if the pupillary centers are discordant, indicating strabismus. The device is also designed to detect ptosis (the pupil is not fully visible to the camera, leaving it unable to record properly, generating a referral report). It has the potential to detect media opacity, but this should be validated with further research. The report can be printed and includes a diagrammatic representation of the eyes showing an estimation of the refractive error, and a graphic presentation of refractive or alignment issues of concern in the child. ${ }^{32}$ There are two software versions (v1.1.51 and v2.0.16) for the same device ${ }^{14}$ with different accuracy indexes for each referral criterion (Table 2 ). ${ }^{1}$

\section{$2 \mathrm{WIN}$}

The 2WIN (Alaska Blind Child Discovery; Adaptica, Padova, Italy) handheld infrared videorefractor is a device that can estimate refractive error and binocular alignment by infrared photoscreening. It has no internal fixation target to avoid proximal accommodation; instead, it uses distant real-world targets as fixation point. It is small, easy to use, and employs several important technologies for a faster and more accurate screening process and efficient record-keeping. ${ }^{6}$

It measures binocular infrared photorefraction and evaluates the gaze direction, ocular alignment, pupil diameter, pupil distance, and the accommodative balance between both eyes. This differs from isotropic refraction, which essentially measures accommodative lag and relates lag value with the eye's refraction. ${ }^{6}$

Refraction is constantly monitored and is based on measures in four different meridians, whereas binocular alignment is maintained using corneal reflexes. Mesopic light is needed for pupils diameter between 4 and $7 \mathrm{~mm}$. The examiner adjusts the measurement distance by focusing while finding the corneal reflexes. Two green circles and a horizontal line appear around the patient pupils. The manufacturer advises that only measurements with enough focus will be recorded and refractive outcomes with a precision of $\pm 0.25 \mathrm{D}$ for power and $1^{\circ}$ for axis will be obtained. Manual averaging of two accurate measurements is the basis of statistical analysis. ${ }^{6}$

\section{Other devices}

Other devices like PR1000 and PR2000 (Topcon, Tokyo, Japan), VPR1 ${ }^{33}$ (Clement Clarke Ltd., Harlow, UK), Vision Research Visiscreen OSS-C photoscreener (Vision Research Corporation, Birmingham, AL, USA), ${ }^{34}$ and SureSight (Welch Allyn) ${ }^{35}$ have been proposed like photorefractors, but there is little information about their performance, accuracy, and utility in amblyopia screening.

\section{Comparison between photoscreener devices}

Due to the different characteristics of the studies and the variables displayed, it is difficult to compare the photoscreeners. Some studies show a single risk factor for amblyopia (anisometropia, ptosis, or strabismus), and other studies show different amblyogenic or strabismus risk factors. Besides, the manufacturer referral criteria are different for each device, making the comparison more difficult. The most used criteria were proposed by the AAPOS, but sometimes, referral criteria are modified. Moreover, accuracy indexes have been compared with different approaches, like the gold-standard (complete exploration with cycloplegic retinoscopy) tabletop binocular photoscreener like Shin-Nippon SRW-5000, ${ }^{26}$ also with tabletop monocular autorefractor like Topcon KR8800 autorefractometer, ${ }^{6}$ or with handheld monocular autorefractor like Retinomax K-plus2. ${ }^{5}$

In general, the data were successfully obtained in $>54 \%$ of children using the Plusoptix S12, ${ }^{35}$ and in $94 \%$ with the MTI. ${ }^{2}$ There was no statistical dependence with the age, but the referral criteria was dependent on the age, ${ }^{3}$ however, older children helped with successful acquisition of the images. ${ }^{2}$ Table 2 shows direct comparison across different studies on measures of accuracy indexes (sensitivity, specificity, positive predictive value, negative predictive value, etc). This comparison should be made with caution because different versions of the instruments, different screening referral criteria, and different examination-positive criteria were used in each study. In addition, when using a composite examinationpositive definition (eg, "astigmatism or hyperopia or myopia or anisometropia"), the same instrument version and same criteria may still yield different specificities and sensitivities for the composite outcome in populations with different relative prevalence of different types of refractive errors. ${ }^{4,19,35}$

It is well known that change in the referral criteria can modify accuracy indexes. Rogers et $\mathrm{al}^{2}$ found similar sensitivities with SureSight and MTI. When using the SureSight manufacturer's criteria, the specificity of the MTI was more than twice that of the SureSight. However, sensitivity calculated using the VIP criteria was lower than that calculated with MTI or SureSight using the manufacturer's criteria. Matta et $\mathrm{al}^{23}$ compared the effectiveness of MTI and Plusoptix S04 photoscreeners using their modified referral criteria. Although both performed well, the study showed Plusoptix S04 to be superior with this modified criterion. Silbert et al ${ }^{18}$ found that the manufacturer's referral criteria for SureSight 
are well suited, with the same sensitivity as Plusoptix A09 but unfortunately with lower specificity. The specificity of SureSight could greatly be increased utilizing the VIP referral criteria, but then the sensitivity will decrease.

No report has been found about sensitivity and specificity of PowerRefractor for amblyopia detection, but it has been demonstrated to be tolerant to eye movements (from $8 \mathrm{~cm}$ toward and $20 \mathrm{~cm}$ away from the correct photorefractor-toeye distance) and changes in background illuminance (from 0.5 to $20 \mathrm{~cd} / \mathrm{m}^{2}$ target luminance). ${ }^{25}$ When the PowerRefractor is compared with tabletop Shin-Nippon SRW-5000 autorefractor, there are no great differences. ${ }^{26}$ Good intersession and intra-session repeatability of these instruments (PowerRefractor or MTI) have been reported (slight for nonexpert examiner and moderate for expert examiner), similar to previously validated open-field autorefractors. ${ }^{19}$ This would indicate that the variability is due to the lack of individual calibration solved in the PowerRefractor $\mathrm{II},{ }^{21}$ but the poor accommodative stimulus of the camera head LEDs, and perhaps the linear range of the change in light gradient across the pupil with different refractive errors, could affect the outcomes. ${ }^{26}$

Whereas the Spot photoscreener provides an estimated refraction in all patients measured, only $75.2 \%$ of patients explored with Plusoptix showed a reliable measurement. ${ }^{13}$ Although the Plusoptix has been demonstrated to be useful in children with high amblyopic risk, its results in strabismus are limited; however, Spot photoscreener was able to measure binocular refraction in larger percentage of patients, especially those with strabismus. ${ }^{13}$

Comparing 2WIN videorefractor with Topcon KR8800 autorefractometer (monocular tabletop), no statistically significant difference has been reported with subjective refraction value obtained in two different sessions (reproducibility), but the reproducibility values achieved with 2 WIN videorefractor have been considerably worse than those achieved with subjective refraction or autorefraction (Topcon KR8800). This lack of reproducibility with 2 WIN probably limits its usefulness as a primary screening device. ${ }^{6}$ Moreover, 2WIN videorefractor has showed lower sensitivity and specificity than Plusoptix S12 photoscreener but similar sensitivity and higher specificity than Spot photoscreener in amblyopia detection. ${ }^{6}$

\section{Discussion}

The Lions Clubs International Foundation programs should be considered a starting point from which vision screening evolves trying to be cost-effective and time-efficient. ${ }^{17}$ The VIP study demonstrated the usefulness of the photoscreener in vision screening in preschoolers. ${ }^{9}$ Commercial photorefractive devices have been available for a long time, with the single purpose of measuring static (unaccommodated) refractive error. For example, MTI photoscreener is used in preschool screening in the US but requires experts to collect data and to assess the image of the pupil crescents. ${ }^{26}$

Wide variation in sensitivity and specificity in amblyopia detection has been reported (Table 2), possibly because of heterogeneous analysis background, heterogeneous or undefined patients, or variable quality of the captured images or photographs. ${ }^{10}$ During the development stages of these devices, the manufacturer must balance sensitivity and specificity to improve the outcomes of screening. ${ }^{1}$

Infrared photoscreeners are effective for early detection of amblyopia, high refractive error measurement, and nonintermittent strabismus screening, ${ }^{32}$ and help reach the percentage of children who did not receive the recommended preschool vision screening and therefore reducing the permanent vision impact of these conditions. ${ }^{20}$

Generally, handheld photoscreeners have been found to underestimate hyperopia and overestimate myopia according to spherical equivalent when their outcomes were compared with cycloplegic retinoscopy. ${ }^{4,5,13}$ Plusoptix and Spot photoscreeners have been found to overestimate astigmatism, ${ }^{35}$ although many studies used the spherical equivalent as it indicated better agreement with cycloplegic retinoscopy. ${ }^{13}$ Refractive measurements correlate significantly with cycloplegic refraction (a strong correlation with Spot photoscreeners and a good correlation with the Plusoptix). ${ }^{13,35}$ For this reason, the estimated refraction is not accepted to be suitable like final prescription, although one study suggests that the Plusoptix A09 may eliminate the need for cycloplegia in early detection of refractive errors in children. ${ }^{30}$ However, the authors concluded that further studies with a larger population having extreme ametropic eyes may be needed to confirm this study result. ${ }^{30}$

The use of photorefractors allows the noninvasive quick measurement of refraction and ocular alignment in both eyes, and they would be of great value in refractive error screening, early detection of amblyopia, and in eye care practice and research. ${ }^{26}$

Great knowledge in eye care is not necessary to operate these instruments, but the ability of the examiner to properly use the photoscreener greatly increases the reliability rate of these devices. ${ }^{7}$ Some studies found variability between operators when performing photorefraction measures, ${ }^{36,37}$ and other authors highlighted the operator experience as an important factor to obtain reliable results. It is estimated that a learning curve of 100 tests to achieve enough experience. ${ }^{2}$ 
However, despite these recommendations, these devices are not a habitual screening method. Most children are not screened, and amblyopia may go unnoticed, perhaps because traditional vision-screening methods have low sensitivity and low specificity. ${ }^{4,22,24}$ However, the traditional eye exam by the eye care practitioner requires a considerable amount of time and experience, and is dependent on the child's understanding and cooperation, and therefore, is relatively difficult to perform with child $<3$ years old. ${ }^{7}$ However, photoscreening can be performed in preverbal and nonverbal children (when a traditional exploration loses reliability) because it is faster (a few seconds) and noninvasive, and does not need child's cooperation and could help in early diagnosis of amblyopia improving the possibility of recovering the visual function of the child. ${ }^{10}$

In conclusion, photoscreening devices are a heterogeneous group of instruments with interest to refractive error screening and early detection of amblyopia, but the differences in previous reports make difficult the comparison between devices. More research that clarifies their utility in clinical practice is necessary.

\section{Acknowledgment}

S Ortiz-Toquero was supported by Junta Castilla y León (Consejeria de Educación) Program: Estrategia Regional de Investigación Científica, Desarrollo Tecnológico e Innovación 2007Y2013, cofunding by Social European Fund.

\section{Disclosure}

The authors report no conflicts of interest in this work.

\section{References}

1. Garry GA, Donahue SP. Validation of Spot screening device for amblyopia risk factors. J AAPOS. 2014;18(5):476-480.

2. Rogers DL, Neely DE, Chapman JB, et al. Comparison of the MTI Photoscreener and the Welch-Allyn SureSight autorefractor in a tertiary care center. J AAPOS. 2008;12(1):77-82.

3. Matta NS, Singman EL, Silbert DI. Performance of the Plusoptix vision screener for the detection of amblyopia risk factors in children. J AAPOS. 2008;12(5):490-492.

4. Yan XR, Jiao WZ, Li ZW, Xu WW, Li FJ, Wang LH. Performance of the Plusoptix A09 photoscreener in detecting amblyopia risk factors in Chinese children attending an eye clinic. PLoS One. 2015;10(6):e0126052.

5. Paff T, Oudesluys-Murphy AM, Wolterbeek R, et al. Screening for refractive errors in children: the plusoptiX S08 and the Retinomax $\mathrm{K}$-plus2 performed by a lay screener compared to cycloplegic retinoscopy. J AAPOS. 2010;14(6):478-483.

6. Ogbuehi KC, Almaliki WH, AlQarni A, Osuagwu UL. Reliability and reproducibility of a handheld videorefractor. Optom Vis Sci. 2015;92(5): 632-641.

7. Salcido AA, Bradley J, Donahue SP. Predictive value of photoscreening and traditional screening of preschool children. J AAPOS. 2005;9(2): 114-120.
8. Dean SE, Povey RC, Reeves J. Assessing interventions to increase compliance to patching treatment in children with amblyopia: a systematic review and meta-analysis. Br J Ophthalmol. 2016;100(2): 159-165.

9. Vision in Preschoolers Study Group. Does assessing eye alignment along with refractive error or visual acuity increase sensitivity for detection of strabismus in preschool vision screening? Invest Ophthalmol Vis Sci. 2007;48(7):3115-3125.

10. Paysse EA, Williams GC, Coats DK, Williams EA. Detection of red reflex asymmetry by pediatric residents using the Brückner reflex versus the MTI photoscreener. Pediatrics. 2001;108(4):E74.

11. Erdurmus M, Yagci R, Karadag R, Durmus M. A comparison of photorefraction and retinoscopy in children. J AAPOS. 2007;11(6):606-611.

12. Miller JM, Lessin HR. Instrument-based pediatric vision screening policy statement. Pediatrics. 2012;130(5):983-986.

13. Peterseim MM, Papa CE, Wilson ME, et al. Photoscreeners in the pediatric eye office: compared testability and refractions on high-risk children. Am J Ophthalmol. 2014;158(5):932-938.

14. Peterseim MM, Papa CE, Wilson ME, et al. The effectiveness of the Spot Vision Screener in detecting amblyopia risk factors. $J$ AAPOS. 2014;18(6):539-542.

15. Ransbarger KM, Dunbar JA, Choi SE, Khazaeni LM. Results of a community vision-screening program using the Spot photoscreener. J AAPOS. 2013;17(5):516-520.

16. Schimitzek T, Lagrèze WA. Accuracy of a new photo-refractometer in young and adult patients. Graefes Arch Clin Exp Ophthalmol. 2005; 243(7):637-645.

17. Donahue SP, Baker JD, Scott WE, et al. Lions Clubs International Foundation Core Four Photoscreening: results from 17 programs and 400,000 preschool children. J AAPOS. 2006;10(1):44-48.

18. Silbert DI, Matta NS, Ely AL. Comparison of SureSight autorefractor and plusoptiX A09 photoscreener for vision screening in rural Honduras. J AAPOS. 2014;18(1):42-44.

19. Mohan KM, Miller JM, Dobson V, Harvey EM, Sherrill DL. Inter-rater and intra-rater reliability in the interpretation of MTI Photoscreener photographs of Native American preschool children. Optom Vis Sci. 2000; 77(9):473-482.

20. Arana Mendez M, Arguello L, Martinez J, et al. Evaluation of the Spot Vision Screener in young children in Costa Rica. J AAPOS. 2015;19(5): 441-444.

21. Jainta S, Jaschinski W, Hoormann J. Measurement of refractive error and accommodation with the photorefractor PowerRef II. Ophthalmic Physiol Opt. 2004;24(6):520-527.

22. Silbert DI, Arnold RW, Matta NS. Comparison of the iScreen and the MTI photoscreeners for the detection of amblyopia risk factors in children. J AAPOS. 2013;17(1):34-37.

23. Matta NS, Singman EL, Silbert DI. Performance of the plusoptiX S04 photoscreener for the detection of amblyopia risk factors in children aged 3 to 5. J AAPOS. 2010;14(2):147-149.

24. Kirk S, Armitage MD, Dunn S, Arnold RW. Calibration and validation of the 2WIN photoscreener compared to the PlusoptiX S12 and the SPOT. J Pediatr Ophthalmol Strabismus. 2014;51(5):289-292.

25. Wolffsohn JS, Hunt OA, Gilmartin B. Continuous measurement of accommodation in human factor applications. Ophthalmic Physiol Opt. 2002;22(5):380-384.

26. Hunt OA, Wolffsohn JS, Gilmartin B. Evaluation of the measurement of refractive error by the PowerRefractor: a remote, continuous and binocular measurement system of oculomotor function. Br J Ophthalmol. 2003;87(12):1504-1508.

27. Seidemann A, Schaeffel F. An evaluation of the lag of accommodation using photorefraction. Vision Res. 2003;43(4):419-430.

28. Vasudevan B, Ciuffreda KJ, Wang B. An objective technique to measure the depth-of-focus in free space. Graefes Arch Clin Exp Ophthalmol. 2006; 244(8):930-937.

29. Arthur BW, Riyaz R, Rodriguez S, Wong J. Field testing of the plusoptiX S04 photoscreener. J AAPOS. 2009;13(1):51-57. 
30. Yilmaz I, Ozkaya A, Alkin Z, Ozbengi S, Yazici AT, Demirok A. Comparison of the Plusoptix A09 and Retinomax K-Plus 3 with retinoscopy in children. J Pediatr Ophthalmol Strabismus. 2015;52(1):37-42.

31. Singman E, Matta N, Tian J, Brubaker A, Silbert D. A comparison of the PlusoptiX S04 and A09 photoscreeners. Strabismus. 2013;21(2): 85-87.

32. Silbert DI, Matta NS. Performance of the Spot vision screener for the detection of amblyopia risk factors in children. J AAPOS. 2014;18(2): $169-172$.

33. Anker S, Atkinson J, Braddick O, et al. Identification of infants with significant refractive error and strabismus in a population screening program using noncycloplegic videorefraction and orthoptic examination. Invest Ophthalmol Vis Sci. 2003;44(2):497-504.
34. Yanovitch T, Wallace DK, Freedman SF, et al. The accuracy of photoscreening at detecting treatable ocular conditions in children with Down syndrome. J AAPOS. 2010;14(6):472-477.

35. Crescioni M, Miller JM, Harvey EM. Accuracy of the Spot and Plusoptix photoscreeners for detection of astigmatism. J AAPOS. 2015;19(5): 435-440.

36. Chat SW, Edwards MH. Clinical evaluation of the Shin-Nippon SRW5000 autorefractor in children. Ophthalmic Physiol Opt. 2001;21(2): 87-100.

37. Williams C, Lumb R, Harvey I, Sparrow JM. Screening for refractive errors with the Topcon PR2000 Pediatric Refractometer. Invest Ophthalmol Vis Sci. 2000;41(5):1031-1037.
Clinical Ophthalmology

\section{Publish your work in this journal}

Clinical Ophthalmology is an international, peer-reviewed journal covering all subspecialties within ophthalmology. Key topics include: Optometry; Visual science; Pharmacology and drug therapy in eye diseases; Basic Sciences; Primary and Secondary eye care; Patien Safety and Quality of Care Improvements. This journal is indexed on

Submit your manuscript here: http://www.dovepress.com/clinical-ophthalmology-journal

\section{Dovepress}

PubMed Central and CAS, and is the official journal of The Society of Clinical Ophthalmology (SCO). The manuscript management system is completely online and includes a very quick and fair peer-review system, which is all easy to use. Visit http://www.dovepress.com/ testimonials.php to read real quotes from published authors. 